\title{
Six-minute walk distance predicts hospitalization in elderly peritoneal dialysis patients: a single-center prospective cohort study
}

Hiroki Yabe ${ }^{1,2^{*}}$ D, Yuto Imoto², Ayaka Onoyama², Sayaka Ito ${ }^{2}$, Kenichi Kono ${ }^{3}$, Yoshifumi Moriyama ${ }^{4}, K^{2}$ eiko Okada ${ }^{5}$, Hirotake Kasuga ${ }^{5}$ and Yasuhiko Ito $^{6}$

\begin{abstract}
Background: The risk of adverse events associated with peritoneal dialysis (PD) in elderly patients has not been thoroughly investigated. The purpose of this study was to assess the association between physical function and hospitalization in elderly PD patients.
\end{abstract}

Methods: This is a single-center prospective observational cohort study. Thirty-three aged patients (74.8 \pm 5.9 years) participated in a 6-min walk distance, short physical performance battery (SPPB), lower extremity muscle strength, and 10-m walk speed. All subjects were followed until hospitalization to the end of the follow-up period.

Results: The 6-min walk distance was $332 \pm 112.5 \mathrm{~m}$; SPPB was 11 (8.3-12) points; the lower extremity muscle strength was $36.6 \pm 9.6 \%$; 10-m walk speed was $1.1 \pm 0.2 \mathrm{~m} / \mathrm{s}$. During the follow-up, 19 patients (57.5\%) were hospitalized. In the Kaplan-Meier survival analysis and log-rank test, a lower 6-min walk distance and PD vintage were significantly associated with hospitalization $(p<0.05)$. After adjustment for PD vintage in Cox proportional analysis, the 6-min walk distance remained associated with hospitalization (95\% confidence interval, 0.98-0.99).

Conclusion: Lower exercise tolerance assessed by the 6-min walk distance was significantly associated with hospitalization in elderly PD patients. Our findings indicate that measurement and intervention of exercise intolerance are essential to predict the clinical outcomes of elderly PD patients.

Trial registration: This study was prospectively registered at inception in the UMIN Clinical Trials Registry under identification number UMIN000038405.

Keywords: Six-minute walk distance, Peritoneal dialysis, Elderly patients, Hospitalization

\footnotetext{
* Correspondence: hiroki-y@seirei.ac.jp

${ }^{1}$ Department of Physical Therapy, School of Rehabilitation Sciences, Seirei

Christopher University, 3453 Mikatahara, Kita-ku, Hamamatsu, Shizuoka 433-8558, Japan

2Department of Rehabilitation, Nagoya Kyoritsu Hospital, 1-172 Hokke,

Nakagawa-ku, Nagoya, Aichi 454-0933, Japan

Full list of author information is available at the end of the article
}

(C) The Author(s). 2021 Open Access This article is licensed under a Creative Commons Attribution 4.0 International License, which permits use, sharing, adaptation, distribution and reproduction in any medium or format, as long as you give appropriate credit to the original author(s) and the source, provide a link to the Creative Commons licence, and indicate if changes were made. The images or other third party material in this article are included in the article's Creative Commons licence, unless indicated otherwise in a credit line to the material. If material is not included in the article's Creative Commons licence and your intended use is not permitted by statutory regulation or exceeds the permitted use, you will need to obtain permission directly from the copyright holder. To view a copy of this licence, visit http://creativecommons.org/licenses/by/4.0/ The Creative Commons Public Domain Dedication waiver (http://creativecommons.org/publicdomain/zero/1.0/) applies to the data made available in this article, unless otherwise stated in a credit line to the data. 


\section{Introduction}

The number of elderly patients with end-stage renal disease (ESRD) and those undergoing peritoneal dialysis (PD) is rising in Japan. In 2018, the average starting age for dialysis was 69.99 years, and this average age has increased yearly [1]. PD for elderly ESRD patients is reaffirming as it allows older patients to undergo dialysis in their own homes. On the other hand, management of various issues in the clinical setting besides access to PD, such as PD-related infection, PD adequacy, nutrition, heart failure, chronic kidney disease (CKD) mineral and bone disorder, assisted PD, geriatric assessment, and social support, also needs to be considered. Risk factors associated with the specific prognosis for elderly PD patients need to be explored, and appropriate intervention for these risk factors to perform PD continuously and stably without adverse events must be determined.

A previous study showed that PD-related peritonitis, infection (except for PD-related peritonitis), cardiac and aortic disease, sudden death, and cerebrovascular disease were causes of death from PD withdrawal [2]. Transfer from PD to in-center hemodialysis (HD) can be related to modality-associated complications, such as peritonitis or tunnel infections, catheter malfunction, and patient factors, such as stroke, worsening vision, or abdominal catastrophes unrelated to PD [3]. The risk of peritonitis, a major factor in PD withdrawal, was associated with the age at PD initiation, diabetes mellitus nephropathy, and the use of a sterile tubing welder device [4]. Prior HD therapy, catheter-related infections, hepatitis $C$ viral infection [5], and hyponatremia [6] were also associated factors for peritonitis.

While these previous studies illustrated the risk factors of PD, the risk in elderly PD patients has not yet been thoroughly investigated, and further investigation of the risk of adverse events specific to elderly PD patients is needed.

One critical risk of CKD and aging is the decline in physical function. CKD leads to the retention of metabolic waste products and a hormonal disturbance that adversely affects multiple target organ systems, including skeletal muscle. Physical performance measures may help assess several risks in CKD patients because previous studies have shown that physical functions in CKD patients affect their prognosis. A previous study showed that the lower measurement of gait speed and the timed up-and-go test was associated with the 3-year all-cause mortality in CKD patients in stages II-IV [7]. In HD patients, lower thigh muscle mass [8], lower extremity muscle strength [9], and exercise capacity [10] were associated with mortality. A previous study on middleaged PD patients also showed that exercise intolerance was significantly associated with all-cause mortality [11]. However, to the best of our knowledge, no study has reported the relationship between physical function and risk factors of prognosis in elderly PD patients.

This study aimed to assess the association of physical function and prognosis in elderly PD patients. We hypothesized that low physical function in elderly PD patients affects their prognosis.

\section{Materials and methods}

\section{Study design and participants}

This is a single-center prospective observational cohort study in the Nephrology outpatients unit of Nagoya Kyoritsu Hospital, Aichi, Japan. Stable ambulatory patients undergoing PD were enrolled from December 1, 2014, to December 1, 2016. Patients were eligible if they met the following criteria: (1) over 65 years old; (2) had been undergoing PD for at least 3 months; and (3) clinically stable. Exclusion criteria included (1) peritonitis or other severe infection needing intravenous antibiotic use, trauma, or operation during the 30 days preceding enrolment; (2) history of stroke or amputation; (3) expectation of PD cessation within 6 months; (4) hospitalization within the previous 3 months; (5) requirement of walking assistance; and (6) impaired cognitive function that would cause difficulty in understanding the evaluation. The ethics committee of Seirei Christopher University approved all procedures performed in this study (Approval No. 16064). Informed consent was obtained from all individual participants included in the study. This study was prospectively registered at inception in the UMIN Clinical Trials Registry under identification number UMIN000038405. All subjects began accruing risk time from the time each physical function was measured. They were followed from hospitalization to the end of the follow-up period (December 31, 2018). The cause of hospitalization was recorded.

\section{Data collection and patient evaluation}

Patients participated in a 6-min walk distance, short physical performance battery (SPPB), lower extremity muscle strength, and 10-m walk speed. The 6-min walk distance was measured by asking the participants to walk as fast as possible along a marked indoor corridor and recording the total distance traveled after $6 \mathrm{~min}$. If the participant could not complete the full 6-min walk, the total distance completed was measured. SPPB is a wellestablished tool to assess the lower extremity physical performance status. SPPB consists of balance tests, gait speed, and chair stand, and the scores range from 0 (worst performance) to 12 (best performance). Lower extremity muscle strength was assessed as the maximal voluntary isokinetic knee extensor strength by a handheld dynamometer ( $\mu$-tasF-1, ANIMA, Japan) twice each in the lower extremities of the participants. The 
strongest of the four trials was considered for analysis. Lower extremity muscle strength was standardized by dividing the participant's body weight. The $10-\mathrm{m}$ walk speed was measured by asking participants to walk at their usual speed over a 10-m course, with the faster of two trials entered for analysis.

The demographic characteristics, such as age, sex, body mass index, PD vintage, automated PD or continuous ambulatory PD, the underlying cause of ESRD, and presence of any comorbid disease, such as diabetes, hypertension, and cardiovascular disease, were collected at enrolment of the study. Data for total protein, serum albumin, blood urea nitrogen, creatinine, sodium, potassium, calcium, phosphorus, hemoglobin, and C-reactive protein levels were obtained from the latest results of physical function in this study.

\section{Statistical analysis}

Data are summarized as mean \pm standard deviation (SD) for normally distributed variables and median (25th to 75th percentiles) for variables with skewed distribution. The normality of the data was examined using the Shapiro-Wilk test. Subjects were divided according to the presence or absence of each adverse event and compared between groups by Student's t-test or the MannWhitney $\mathrm{U}$ test, depending on data distribution.

Subjects were divided into two groups based on the reference values of the physical function indicated in the previous studies. The adopted reference values were as follows: 6-min walk distance, $<350 \mathrm{~m}$ [7]; short physical performance battery, $<8$ points [12]; lower extremity muscle strength, $<40 \%$ [9]; and the 10-m walk speed, $<1$ $\mathrm{m} / \mathrm{s}$ [13]. Age and PD vintage were divided into two groups based on the median values $(<75$ years and $<24$ months, respectively). The relationship between the hospitalization and each measurement value used for the grouping was studied using the Kaplan-Meier analysis and log-rank test to estimate unadjusted cumulative survival during follow-up and to assess the statistical differences in survival. The Cox proportional hazard regression model was used to estimate adjusted hazard ratios (HR) and 95\% confidence interval (CI) for hospitalization associated with each physical performance measure. Adjustment covariates were selected before analysis to assess the relationship between confounding factors. The covariates added variables that were significant in the log-rank test. All tests were assessed at the $\mathrm{p}<0.05$ level of significance. All statistical analyses were performed using IBM SPSS Statistics for Windows, ver. 21 (IBM Corp., Armonk, NY, USA).

\section{Results}

Thirty-seven patients were screened for the study. Of these 37 patients, three patients refused to participate in the study, and one patient was excluded due to medical reasons (Fig. 1). Consequently, a total of 33 patients were enrolled in the study (23 men and 10 women; mean age, $74.8 \pm 5.9$ years; median PD vintage value, 24 (13-42) months) (Table 1). The 6-min walk distance was $332.3 \pm 112.5 \mathrm{~m}$, SPPB was $11(8.3-12)$ points, the lower extremity muscle strength was $36.6 \% \pm 9.6 \%$, and the $10-\mathrm{m}$ walk speed was $1.1 \pm 0.2 \mathrm{~m} / \mathrm{s}$. Fifteen $(45.5 \%)$

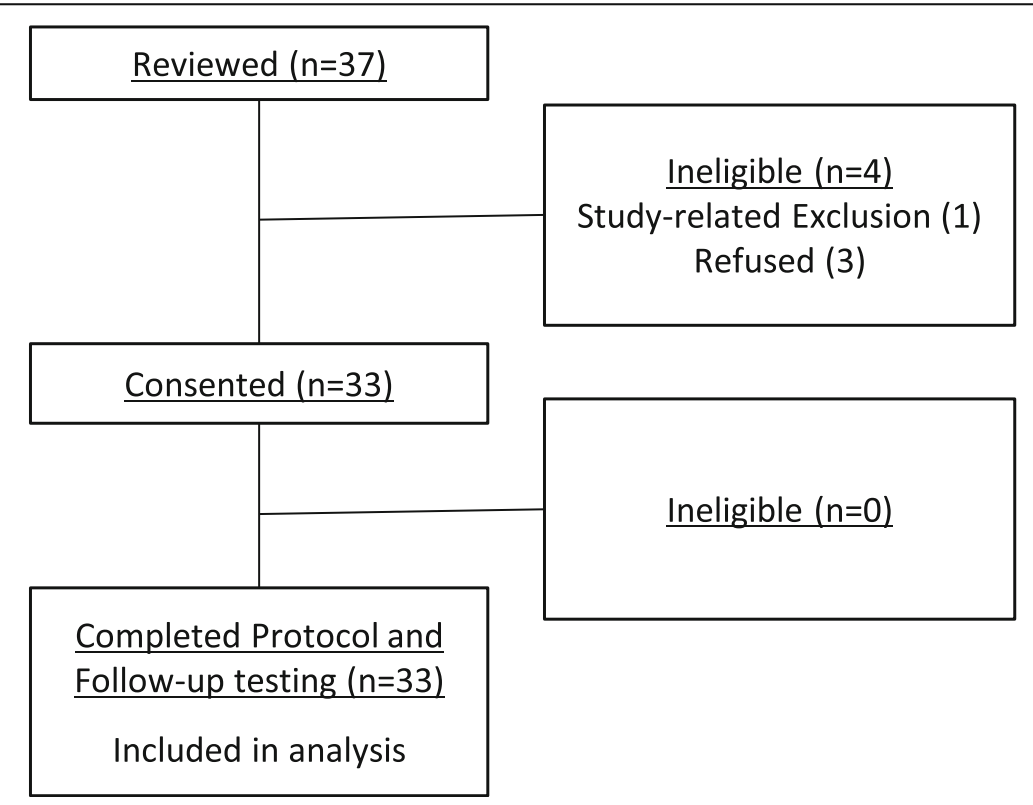

Fig. 1 Flow of participants. Patients were enrolled from December 1, 2014, to December 1, 2016, and they were followed for a mean of $24.3 \pm 15.3$ months until December 31, 2018 
Table 1 Patient characteristics

\begin{tabular}{ll}
\hline Variable & Mean \pm SD \\
\hline Age (year) & $74.8 \pm 5.9$ \\
Sex (male/female) & $23 / 10$ \\
BMI (kg/m²) & $22.5 \pm 2.8$ \\
PD vintage (month) & $24(13-42)$ \\
Type of PD (APD/CAPD, $n)$ & \\
Primary renal disease ( $\mathrm{n}(\%))$ & \\
$\quad$ Diabetic nephrology & $13(39.4)$ \\
$\quad$ Chronic glomerulonephritis & $9(27.3)$ \\
$\quad$ Nephrosclerosis & $6(18.2)$ \\
$\quad$ Others & $5(15.2)$ \\
Comorbidity (n (\%)) & \\
$\quad$ Hypertension & $32(97)$ \\
$\quad$ Diabetes mellitus & $21(63.6)$ \\
$\quad$ Cardiovascular disease & $10(30.3)$ \\
\hline
\end{tabular}

The values are expressed as mean \pm SD or median (25th to 75 th percentiles)

patients in the 6-min walk distance, 19 (57.6\%) patients in SPPB, 15 (45.5\%) patients in lower extremity muscle strength, and $16(48.5 \%)$ patients in 10-m walk speed were not more than reference respectively (cases, \%) (Table 2).

The average follow-up period was $24.3 \pm 15.3$ months. A total of 19 patients (57.5\%) were hospitalized during follow-up (Table 3). Five (15.2\%) patients were diagnosed with peritonitis, including two hospitalized patients. The group hospitalized during follow-up was significantly older (78.1 \pm 5.8 vs. $71.6 \pm 4.3$ years) and had a longer PD vintage [36 (18-76.5) vs. 20 (10.5-29.5) months], a lower 6 -min walk distance $(270.9 \pm 97.6$ vs. $386.1 \pm 102.2 \mathrm{~m})$, and lower SPPB scores [10 (7-10) vs. $12(10.3-12)]$ than did the group without hospitalization ( $\mathrm{p}<0.05$, Table 4) (hospitalized vs. not hospitalized group, respectively).

In the Kaplan-Meier survival analysis and log-rank test, a lower 6-min walk distance was significantly associated with hospitalization (Fig. 2a, p<0.05). A lower SPPB (Fig. 2b, $\mathrm{p}=0.06$ ) tended to be associated with hospitalization. Lower extremity muscle strength (Fig. 2c), the 10-m walk speed (Fig. 2d), and age (Fig. 2e) were not significantly associated with hospitalization
Table 3 Causes and number of hospitalizations

\begin{tabular}{ll}
\hline Cause & Cases \\
\hline Cerebral infarction & 3 \\
Peritonitis & 2 \\
Fever of unknown origin & 2 \\
Bronchitis & 2 \\
Fall/cervical myelopathy & 2 \\
Heart failure & 1 \\
Heart failure + pneumonia & 1 \\
Pneumonia & 1 \\
Acute myocardial infarction & 1 \\
Tunnel infection & 1 \\
Rotary vertigo & 1 \\
Anorexia, hyponatremia & 1 \\
Blood glucose control & 1 \\
\hline
\end{tabular}

$(\mathrm{p}>0.05)$. PD vintage was significantly associated with hospitalization (Fig. 2f, p<0.05). After adjustment for PD vintage in Cox proportional analysis, the 6-min walk distance remained associated with hospitalization (Table 5, HR 0.96, 95\% CI 0.98-0.99).

\section{Discussion}

Our study found that lower scores of 6-min walk distance were associated with hospitalization after adjustment for PD vintage, suggesting that simple objective exercise tolerance measures might be useful for risk stratification of elderly patients with PD. This result suggests that the lower exercise tolerance may represent important therapeutic targets in this population. Although the number of elderly patients receiving $\mathrm{PD}$ is considerably fewer than that of elderly patients receiving HD, the importance of PD for elderly patients is increasing. Although the sample size of our study was relatively small, the study findings may have clinical implications for PD.

With an increase in the aging population, the proportion of HD patients is increasing globally, and the importance of PD for elderly CKD patients is also increasing. A significant increase in the average age of people undergoing dialysis was observed in almost all 12

Table 2 Results of physical function measurements

\begin{tabular}{llll}
\hline & Measurement value & Reference value & Not more than reference (cases, \%) \\
\hline 6-min walk distance $(\mathrm{m})$ & $332.3 \pm 112.5$ & 350 & $15(45.5)$ \\
SPPB (points) & $11(8.3-12)$ & 8 & $19(57.6)$ \\
Lower extremity muscle strength $(\%)$ & $36.6 \pm 9.6$ & 40 & $15(45.5)$ \\
$10-\mathrm{m}$ walk speed $(\mathrm{m} / \mathrm{s})$ & $1.1 \pm 0.2$ & 1.0 & $16(48.5)$ \\
\hline
\end{tabular}


Table 4 Result of between group comparison

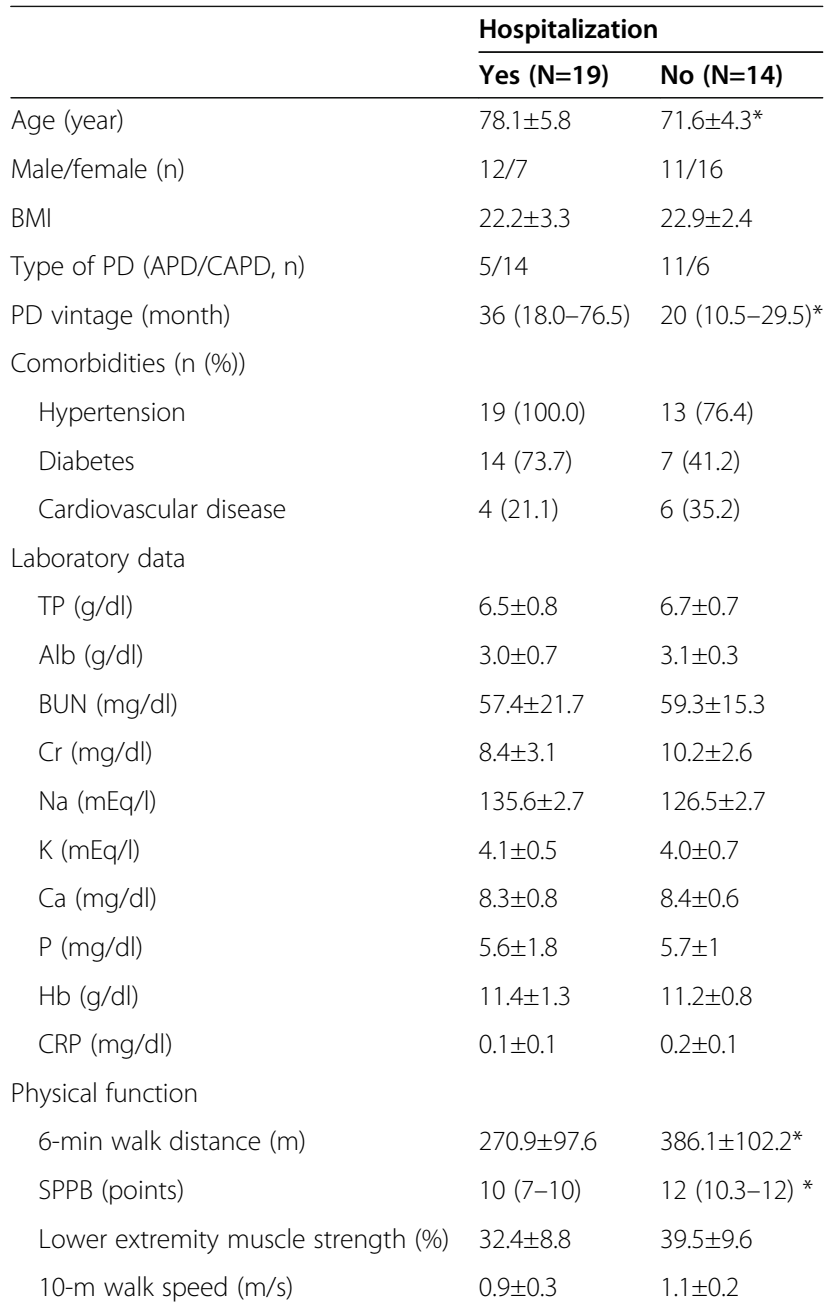

$B M I$ body mass index, $T P$ total protein, Alb albumin, BUN blood urea nitrogen, $\mathrm{Cr}$ creatinine, $\mathrm{Na}$ natrium, $\mathrm{K}$ potassium, $\mathrm{Ca}$ calcium, $P$ phosphorus, $\mathrm{Hb}$ hemoglobin, CRP C-reactive protein levels, SPPB short physical performance battery

The values are expressed as mean \pm SD or median (25th to 75 th percentiles)

* $p<0.05$ according to Student's $t$ and Mann-Whitney tests

nations included in the Dialysis Outcomes and Practice Patterns Study, an international cohort study [14]. PD may be suitable for elderly CKD patients because it has a low circulatory load and may maintain residual renal function. Additionally, PD allows continued dialysis at home. Brown et al. showed in his review article that many patients tolerate HD and transport to and from an HD unit poorly. However, in most parts of the world, older patients are preferentially placed on hospital-based $\mathrm{HD}$ and are considered unsuitable for a home-based self-care dialysis [15]. Physical function declines as renal failure progresses in elderly patients with CKD. These processes are accelerated in elderly dialysis patients due to the uremic condition and aging. Therefore, evaluation of an elderly patient's physical function is important to determine continuous PD at home.
Previous studies have shown that assessment of physical function such as 6-min walk distance and SPPB were useful prognostic indicators in a population other than PD. A study showed that a 6-min walk distance was significantly associated with a higher risk of mortality in patients with CKD stages II-IV [7]. Moreover, in the study of middle-aged PD patients [11], the 6-min walk distance could predict all-cause mortality and technique failure. Lower SPPB scores were significantly associated with a higher risk of hospitalization in older patients [12]. A study in patients undergoing hemodialysis showed no change in frail patients from an SPPB score of $\leq 6$ to $>6$ points and a $29.3 \%$ shift in non-frail patients from an SPPB score of $>6$ to $\leq 6$ points after a 12-month follow-up [16]. Our study results are in line with these previous studies, indicating that a 6 -min walk distance may be a useful predictor in older PD patients. However, our study did not show the relationship between hospitalization and lower extremity muscle strength and 10-m walking speed. In CKD patients not treated with dialysis, each $0.1 \mathrm{~m} / \mathrm{s}$ decrease in gait speed was associated with a $26 \%$ higher risk of death in a previous study [7]. In patients undergoing hemodialysis, lower extremity muscle strength [9] and walking speed [17] were significantly associated with mortality. These variables should be confirmed by additional subjects and/or observation periods, as previous studies have reported these as good predictors of prognosis.

The 6-min walk distance is a useful measurement value that reflects exercise tolerance, has good reliability, is inexpensive, and is easily applicable. Exercise tolerance is generally measured by cardiopulmonary exercise testing (CPX). Patients with peak oxygen uptake (peak $\mathrm{VO}_{2}$ ) that indicate exercise tolerance by CPX exhibited significantly better survival than did those with lower HD values; this suggests that exercise capacity is a strong predictor of survival in dialysis patients [10]. However, compared with the 6-min walk distance, CPX is both expensive and time-consuming. In addition, approximately $30 \%$ of patients with heart failure cannot perform a maximal symptom-limited exercise test or tolerate the tight mask used for breath-by-breath gas analysis [18]. On the other hand, previous studies have shown that the 6-min walk distance value is associated with peak $\mathrm{VO}_{2}$ in patients with chronic heart failure [19] and HD patients [20], and has good reliability and reproducibility [21].

Exercise tolerance measured by the 6-min walk distance may be associated with a prognosis that is affected by several factors, such as age and uremia. PD and HD patients appear to be at a similar risk of developing all geriatric syndromes [22]. A significant consequence of skeletal muscle loss is skeletal muscle dysfunction, which is associated with impaired mobility and reduced physical performance in patients with CKD. Previous studies 

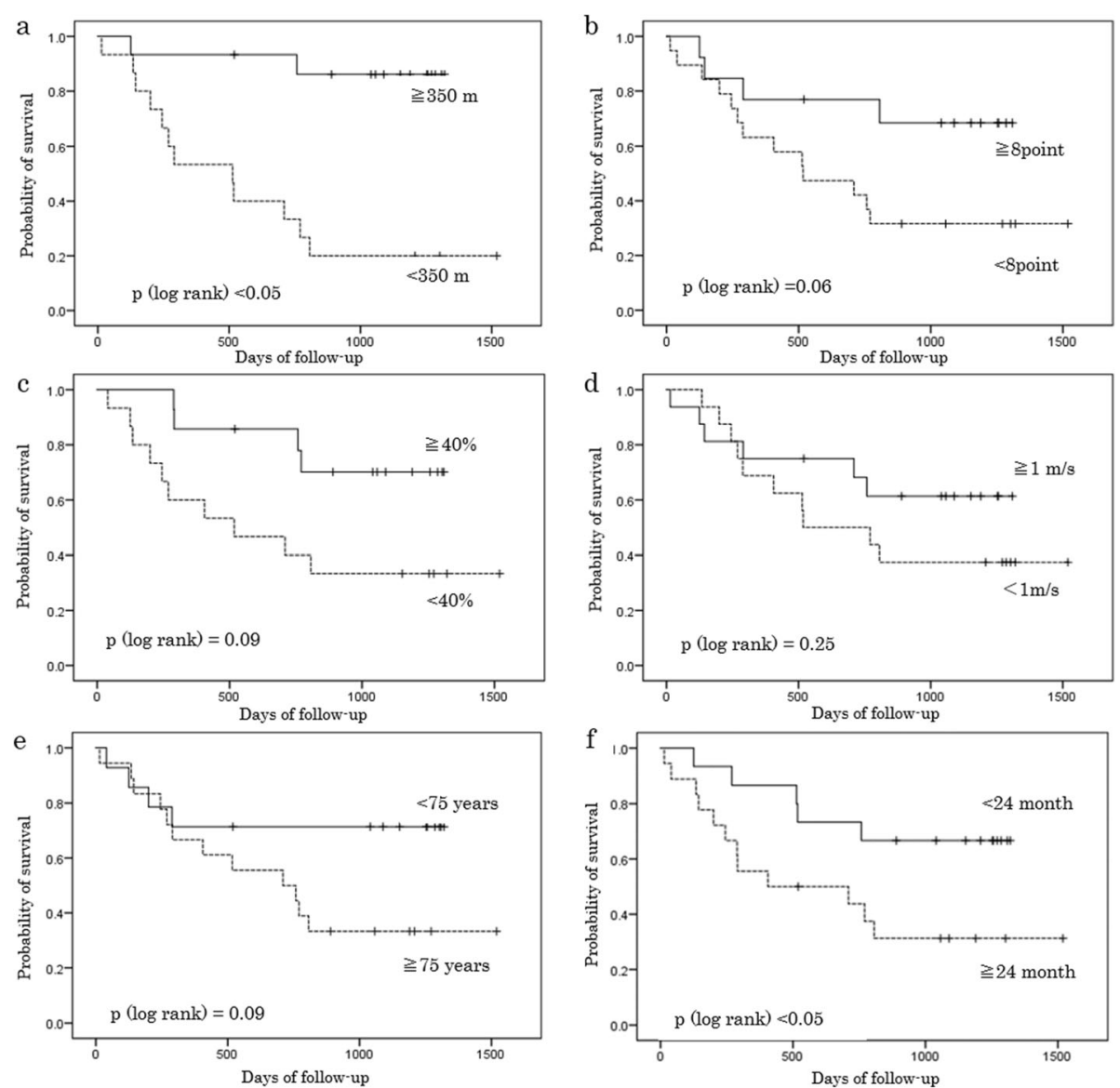

Fig. 2 Kaplan-Meier analysis with patients divided into two groups based on the reference values of the physical function. A lower 6-min walk distance and PD vintage were significantly associated with hospitalization. PD, peritoneal dialysis

have shown that patients with CKD have low muscle mass [23], reduced muscle strength [24], and exercise intolerance [25]. Additional previous studies have shown that muscle strength, balance function, and walking capacity in patients with CKD stages II to $\mathrm{V}$ decreased as the CKD stage progressed [26]. In a separate study, elderly PD patients had lower scores in the chair stand test [27], muscle strength, and exercise tolerance [28] than did healthy controls. In PD patients aged 65 years and older (average $76.2 \pm 7.5$ years), $69 \%$ of subjects were unable to stand from a chair without the use of their arms, and $51 \%$ of subjects had abnormal timed up-andgo test scores. Only $11 \%$ of patients were fully independent for activities of daily living and instrumental activities of daily living [29]. Exercise capacity is determined

Table $\mathbf{5}$ Result of Cox proportional analysis

\begin{tabular}{lllll}
\hline & $\boldsymbol{\beta}$ & $\mathbf{p}$ & $\mathbf{H R}$ & $\mathbf{9 5 \%} \mathbf{C l}$ \\
\hline 6-min walk distance & -0.01 & $<0.05$ & 0.96 & $0.98-0.99$
\end{tabular}

$\mathrm{PD}$ vintage was included as independent variable

$\beta$ partial regression coefficient, $H R$ hazard ratio, $\mathrm{Cl}$ confidence interval by not only cardiovascular function and skeletal muscle mass and function, but also diabetes [30], hypertension [31], hemoglobin concentration, serum albumin [10], age, diastolic blood pressure, and left ventricular ejection fraction [11]. The detailed mechanism is unknown; however, 6-min walk distance would represent an integrated measure of these multiple variables in ESRD. Therefore, the 6-min walk distance may relate to outcomes, such as hospitalization in elderly PD patients.

This study has some limitations. First, because this study had a small sample size, we could not adjust for other confounding factors, such as sex, cardiac function, laboratory data, and comorbidities. The condition of the peripheral artery measured by the ankle brachial index or the toe brachial pressure index may also be associated with adverse events. Second, selection bias might exist due to the long period of enrolment, which included a 22-month interval between enrolment of the first patient and that of the last patient. Since elderly peritoneal dialysis patients are rare, we increased the number of subjects by setting a long enrolment period. Third, the generalizability of the study findings is limited. 
Generally, the sample size should be calculated on the basis of the statistical power; however, this study only included patients from the institution, who may not be representative of the entire population of elderly patients receiving PD. Nevertheless, our findings might be of significant value in the clinical area of nephrology. We could not observe full prognoses, such as death and peritonitis in our study participants, and an investigation with more participants and a more extended follow-up period is needed.

\section{Conclusion}

Lower exercise tolerance measured by 6 -min walk distance was significantly associated with hospitalization in elderly PD patients. Our findings indicate that measurement and intervention of exercise intolerance are important to predict the clinical outcomes of elderly PD patients.

\section{Abbreviations}

CPX: Cardiopulmonary exercise testing; Cl: Confidence interval; CKD: Chronic kidney disease; ESRD: End-stage renal disease; HD: Hemodialysis; HR: Hazard ratios; PD: Peritoneal dialysis; SD: Standard deviation; SPPB: Short physical performance battery

\section{Acknowledgements}

Not applicable.

\section{Authors' contributions}

HY conceived the study, participated in the study design and coordination, and wrote the first draft of the manuscript. KK and Yl participated in the study design and performed the statistical analyses. $\mathrm{Yl}, \mathrm{SI}$, and AO performed the study measurements. YM integrated the research data measurements as the measurement manager. $\mathrm{HK}$ and $\mathrm{KO}$ treated the patients and recorded clinical findings as the principal doctors. The authors read and approved the final manuscript.

\section{Funding}

This study did not receive any funding.

\section{Availability of data and materials}

The datasets used and/or analyzed during the current study are available from the corresponding author on reasonable request.

\section{Declarations}

\section{Ethics approval and consent to participate}

All procedures performed in studies involving human participants were in accordance with the ethical standards of the Seirei Christopher University (IRB approval number 16064) and with the 1964 Helsinki Declaration and its later amendments or comparable ethical standards.

\section{Consent for publication}

Informed consent was obtained from all individual participants included in the study.

\section{Competing interests}

The co-author has received Honoraria from Baxter and research funding from Kyowa-Kirin, Chugai, and Baxter.

\section{Author details}

'Department of Physical Therapy, School of Rehabilitation Sciences, Seirei Christopher University, 3453 Mikatahara, Kita-ku, Hamamatsu, Shizuoka 433-8558, Japan. '2Department of Rehabilitation, Nagoya Kyoritsu Hospital, 1-172 Hokke, Nakagawa-ku, Nagoya, Aichi 454-0933, Japan. ${ }^{3}$ Department of Physical Therapy, School of Health Sciences at Narita, International University of Health and Welfare, 4-3, Kozunomori, Narita, Chiba 286-8686, Japan. ${ }^{4}$ Department of Wellness Center, Nagoya Kyoritsu Hospital, 1-172 Hokke, Nakagawa-ku, Nagoya, Aichi 454-0933, Japan. ${ }^{5}$ Department of Nephrology, Nagoya Kyoritsu Hospital, 1-172 Hokke, Nakagawa-ku, Nagoya, Aichi 454-0933, Japan. ${ }^{6}$ Department of Nephrology and Rheumatology, Aichi Medical University, 1-1 Yazakokarimata, Nagakute, Aichi 480-1195, Japan.

Received: 18 March 2021 Accepted: 3 June 2021

Published online: 16 June 2021

\section{References}

1. Nitta K, Abe M, Masakane I, Hanafusa N, Taniguchi M, Hasegawa T, et al. Annual dialysis data report 2018, JSDT Renal Data Registry: dialysis fluid quality, hemodialysis and hemodiafiltration, peritoneal dialysis, and diabetes. Ren Replace Ther. 2020;6(1):1-6.

2. Mizuno M, Ito Y, Suzuki Y, Sakata F, Saka Y, Hiramatsu T, et al. Recent analysis of status and outcomes of peritoneal dialysis in the Tokai area of Japan: the second report of the Tokai peritoneal dialysis registry. Clin Exp Nephrol. 2016;20(6):960-71. https://doi.org/10.1007/s10157-016-1249-9.

3. Bieber SD, Mehrotra R. Patient and technique survival of older adults with ESRD treated with peritoneal dialysis. Perit Dial Int. 2015;35(6):612-7. https:// doi.org/10.3747/pdi.2015.00050.

4. Nishina M, Yanagi H, Kakuta T, Endoh M, Fukagawa M, Takagi A. A 10-year retrospective cohort study on the risk factors for peritoneal dialysis-related peritonitis: a single-center study at Tokai University Hospital. Clin Exp Nephrol. 2014;18(4):649-54. https://doi.org/10.1007/s10157-013-0872-y.

5. Ozisik L, Ozdemir FN, Tanriover MD. The changing trends of peritoneal dialysis related peritonitis and novel risk factors. Ren Fail. 2015;37(6):102732. https://doi.org/10.3109/0886022X.2015.1052952.

6. Wang HH, Huang CH, Kuo MC, Lin SY, Hsu CH, Lee CY, et al. Microbiology of peritoneal dialysis-related infection and factors of refractory peritoneal dialysis related peritonitis: a ten-year single-center study in Taiwan. J Microbiol Immunol Infect. 2019;52(5):752-9. https://doi.org/10.1016/j.jmii.201 8.10.013.

7. Roshanravan B, Robinson-Cohen C, Patel KV, Ayers E, Littman AJ, de Boer $\mathbf{H}$, et al. Association between physical performance and all-cause mortality in CKD. J Am Soc Nephrol. 2013;24(5):822-30. https://doi.org/10.1681/ASN.2012 070702.

8. Fukasawa $\mathrm{H}$, Kaneko M, Niwa $\mathrm{H}$, Matsuyama T, Yasuda $\mathrm{H}$, Kumagai $\mathrm{H}$, et al. Lower thigh muscle mass is associated with all-cause and cardiovascular mortality in elderly hemodialysis patients. Eur J Clin Nutr. 2017;71(1):64-9. https://doi.org/10.1038/ejcn.2016.186.

9. Matsuzawa R, Matsunaga A, Wang G, Yamamoto S, Kutsuna T, Ishii A, et al. Relationship between lower extremity muscle strength and all-cause mortality in Japanese patients undergoing dialysis. Phys Ther. 2014;94(7): 947-56. https://doi.org/10.2522/ptj.20130270.

10. Sietsema KE, Amato A, Adler SG, Brass EP. Exercise capacity as a predictor of survival among ambulatory patients with end-stage renal disease. Kidney Int. 2004;65(2):719-24. https://doi.org/10.1111/j.1523-1755.2004.00411.x.

11. Shi Y, Zheng D, Zhang $L, Y u Z$, Yan H, Ni Z, et al. Six-minute walk test predicts all-cause mortality and technique failure in ambulatory peritoneal dialysis patients. Nephrology (Carlton). 2017;22(2):118-24. https://doi.org/1 $0.1111 /$ nep. 12726

12. Volpato S, Cavalieri M, Sioulis F, Guerra G, Maraldi C, Zuliani G, et al. Predictive value of the short physical performance battery following hospitalization in older patients. J Gerontol A Biol Sci Med Sci. 2011;66:89_ 96.

13. Roshanravan B, Gamboa J, Wilund K. Exercise and CKD: skeletal muscle dysfunction and practical application of exercise to prevent and treat physical impairments in CKD. Am J Kidney Dis. 2017;69(6):837-52. https:// doi.org/10.1053/j.ajkd.2017.01.051.

14. Canaud B, Tong L, Tentori F, Akiba T, Karaboyas A, Gillespie B, et al. Clinical practices and outcomes in elderly hemodialysis patients: results from the Dialysis Outcomes and Practice Patterns Study (DOPPS). Clin J Am Soc Nephrol. 2011;6(7):1651-62. https://doi.org/10.2215/CJN.03530410.

15. Brown EA, Bargman JM, Li PK. Managing older patients on peritoneal dialysis. Perit Dial Int. 2015;35(6):609-11. https://doi.org/10.3747/pdi.2015.001 59.

16. Ortega-Pérez de Villar $L$, Martínez-Olmos FJ, Junqué-Jiménez A, AmerCuenca JJ, Martínez-Gramage J, Mercer T, et al. Test-retest reliability and minimal detectable change scores for the short physical performance 
battery, one-legged standing test and timed up and go test in patients undergoing hemodialysis. Plos One. 2018;13:e0201035.

17. Abe Y, Matsunaga A, Matsuzawa R, Kutsuna T, Yamamoto S, Yoneki K, et al. Determinants of slow walking speed in ambulatory patients undergoing maintenance hemodialysis. Plos One. 2016;11(3):e0151037. https://doi.org/1 0.1371/journal.pone.0151037.

18. Arslan S, Erol MK, Gundogdu F, Sevimli S, Aksakal E, Senocak H, et al. Prognostic value of 6-minute walk test in stable outpatients with heart failure. Heart Inst J. 2007:34:166-9.

19. Cahalin LP, Mathier MA, Semigran MJ, Dec GW, DiSalvo TG. The six-minute walk test predicts peak oxygen uptake and survival in patients with advanced heart failure. Chest. 1996;1 10(2):325-32. https:/doi.org/10.1378/ chest.110.2.325.

20. Kohl LM, Signori LU, Ribeiro RA. Prognostic value of the six-minute walk test in endstage renal disease life expectancy: a prospective cohort study. Clinics. 2012;67(6):581-6. https://doi.org/10.6061/clinics/2012(06)06.

21. Sequra-Orti E, Martinez-Olmos FJ. Test-retest reliability and minimal detectable change scores for sit-to-stand-to-sit tests, the six-minute walk test, the one-leg heel-rise test, and handgrip strength in people undergoing hemodialysis. Phys Ther. 2011;91(8):1244-52. https://doi.org/10.2522/ptj.201 00141.

22. Jassal SV. Geriatric Assessment, falls and rehabilitation in patients starting or established on peritoneal dialysis. Perit Dial Int. 2015;35(6):630-4. https://doi. org/10.3747/pdi.2014.00342.

23. McIntyre CW, Selby NM, Sigrist M, Pearce LE, Mercer TH, Naish PF. Patients receiving maintenance dialysis have more severe functionally significant skeletal muscle wasting than patients with dialysis-independent chronic kidney disease. Nephrol Dial Transplant. 2006;21(8):2210-6. https://doi.org/1 0.1093/ndt/gfl064

24. Fahal IH, Bell GM, Bone JM, Edwards RH. Physiological abnormalities of skeletal muscle in dialysis patients. Nephrol Dial Transplant. 1997;12(1):11927. https://doi.org/10.1093/ndt/12.1.119.

25. Johansen KL. Physical functioning and exercise capacity in patients on dialysis. Adv Ren Replace Ther. 1999;6(2):141-8. https://doi.org/10.1016/S1 073-4449(99)70032-4.

26. Hiraki K, Yasuda T, Hotta C, Izawa KP, Morio Y, Watanabe S, et al. Decreased physical function in pre-dialysis patients with chronic kidney disease. Clin Exp Nephrol. 2013;17(2):225-31. https://doi.org/10.1007/s10157-012-0681-8.

27. Cupisti A, D'Alessandro C, Finato V, Del Corso C, Catania B, Caselli GM, et al. Assessment of physical activity, capacity and nutritional status in elderly peritoneal dialysis patients. BMC Nephrol. 2017;18(1):180. https://doi.org/1 0.1186/s12882-017-0593-7.

28. Painter PL, Agarwal A, Drummond M. Physical function and physical activity in peritoneal dialysis patients. Perit Dial Int. 2017;37(6):598-604. https://doi. org/10.3747/pdi.2016.00256.

29. Ulutas O, Farragher J, Chiu E, Cook WL, Jassal SV. Functional disability in older adults maintained on peritoneal dialysis therapy. Perit Dial Int. 2016; 36(1):71-8. https://doi.org/10.3747/pdi.2013.00293.

30. Estacio RO, Regensteiner JG, Wolfel EE. The association between diabetic complications and exercise capacity in NIDDM patients. Diabetes Care. 1998; 21(2):291-5. https://doi.org/10.2337/diacare.21.2.291.

31. Lim PO, MacFadyen RJ, Clarkson PB. Impaired exercise tolerance in hypertensive patients. Ann Intern Med. 1996;124(1_Part_1):41-55. https:// doi.org/10.7326/0003-4819-124-1_Part_1-199601010-00008.

\section{Publisher's Note}

Springer Nature remains neutral with regard to jurisdictional claims in published maps and institutional affiliations.

Ready to submit your research? Choose BMC and benefit from:
- fast, convenient online submission
- thorough peer review by experienced researchers in your field
- rapid publication on acceptance
- support for research data, including large and complex data types
- gold Open Access which fosters wider collaboration and increased citations
- maximum visibility for your research: over 100M website views per year
At BMC, research is always in progress.
Learn more biomedcentral.com/submissions

